\title{
Utilization of Temperature-Sweeping Capacitive Techniques to Evaluate Band-Gap Defect Densities in Photovoltaic Perovskites
}

Osbel Almora, ${ }^{* 1,2}$ Marisé García-Batlle ${ }^{1}$ and Germà Garcia-Belmonte ${ }^{* 1}$

${ }^{1}$ Institute of Advanced Materials (INAM), Universitat Jaume I, 12006 Castelló, Spain

${ }^{2}$ Institute of Materials for Electronics and Energy Technology (i-MEET), FriedrichAlexander-Universität Erlangen-Nürnberg (FAU), 91058 Erlangen, Germany \& Erlangen Graduate School in Advanced Optical Technologies (SAOT), FAU, 91052 Erlangen, Germany

*Email: osbel.almora@fau.de, garciag@uji.es

\begin{abstract}
Capacitive techniques, routinely used for solar cell parameter extraction, probe the voltage-modulation of the depletion layer capacitance isothermally as well as under varying temperature. Also defect states within the semiconductor band-gap respond to such stimuli. Although extensively used, capacitive methods have found difficulties when applied to elucidate bulk defect bands in photovoltaic perovskites. This is so because perovskite solar cells (PSCs) actually exhibit some intriguing capacitive features hardly connected to electronic defect dynamics. The commonly reported excess capacitance observed at low frequencies is originated by outer interface mechanisms and has a direct repercussion on the evaluation of band-gap defect levels. Starting by updating previous observations on Mott-Schottky (MS) analysis in PSCs, it is discussed how the thermal admittance spectroscopy (TAS) and the deep level transient spectroscopy (DLTS) characterization techniques present spectra with overlapping or even "fake" peaks caused by the mobile ion-related, interfacial excess capacitance. These capacitive techniques, when used uncritically, may be misleading and produce wrong outcomes.
\end{abstract}


KEYWORDS: Perovskite solar cells; band-gap trap levels; capacitance; impedance spectroscopy

\section{TOC Graphic:}

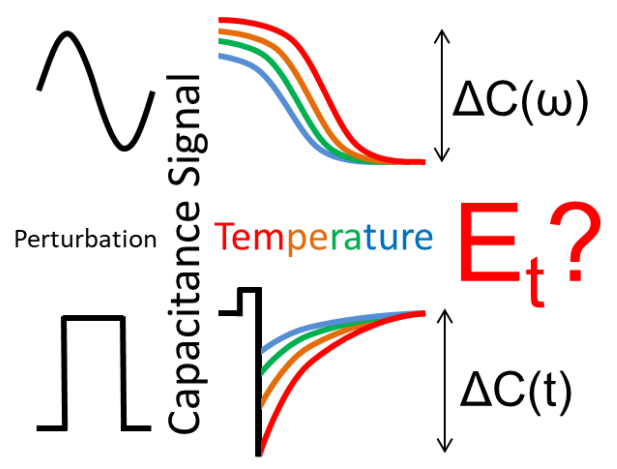


The capacitive characterization procedures of trap levels within the band-gap in active semiconductors composing photovoltaic cells are well-established. ${ }^{1-3}$ These techniques allow extracting relevant information about defect levels influencing solar cell operation and can be used to understand and optimize devices. Consequently, they have been recently applied to elucidate defect densities and related parameters as the activation energy in perovskite solar cells (PSCs), ${ }^{4-7}$ which is maybe the most attractive photovoltaic technology during the last few years due to its high efficiency reports and easy manufacturing processes.$^{8-9}$ Defect levels located at the absorber bulk or at outer interfaces regulate the recombination, doping and transport modes in solar cells, and thus the overall device performance, with a particular characteristic effect in the capacitive response. ${ }^{10-11}$ Accordingly, and in relation with our previous observations on Mott-Schottky (MS) analysis in PSCs, ${ }^{12}$ we survey on the reliability of using temperature-sweeping techniques like the thermal admittance spectroscopy (TAS) and the deep level transient spectroscopy (DLTS) for characterizing trap levels in PSCs.

The standard capacitive technique for determining defect densities and spatial distributions within the semiconductor absorbers is the MS analysis. ${ }^{13}$ Its basis lies on the assumption that a depletion layer is formed in the vicinity of the contact between doped semiconductors and/or metals. A depletion capacitance $C_{d l}=\varepsilon \varepsilon_{0} / w$ (being $\varepsilon$ the dielectric constant and $\varepsilon_{0}$ the vacuum permittivity) is associated with the modulation of the depletion layer width $w$ by an $a c$ perturbation $\tilde{V}$ at a given $d c$ bias voltage $V$ (ac and $d c$ stand for alternate and direct currents, respectively), in such a way that in the one-sided abrupt $p-n$ junction case it is approximated as

$$
C_{d l}=\sqrt{\frac{q \varepsilon \varepsilon_{0} N}{2\left(V_{b i}-V\right)}}
$$


Here $q$ is the elementary charge, $V_{b i}$ corresponds to the built-in voltage and $N$ accounts for the concentration of fixed ionized defects in the space charge region which defines the conductivity of the layer. Figure 1 illustrates typical MS plot representations, i.e. $C^{-2}(V)$, where the evaluation of $N$ and $V_{b i}$ can be taken from the slope and voltage intercept of the linear behaviors, specifically in Figure 1a,c. Note that Equation (1) can be modified depending on the particularities of the junction, meaning that $N, V_{b i}$ and even the power of the expression (0.5 square root) are effective/rough approximate values. ${ }^{14-15}$ For instance, for a $p-i-n$ junction instead of $V_{b i}$ the MS plot voltage intercept results in $V_{b i, w i}=V_{b i}+V_{w i}$ with the voltage drop $V_{w i}=q N w_{i}^{2} / 2 \varepsilon \varepsilon_{0}$ in the intrinsic layer $w_{i}$. Illustratively, for typical $p-i-n$ perovskite devices $w_{i} \approx 400 \mathrm{~nm}$ so $V_{w i}$ can be neglected if $N<10^{15} \mathrm{~cm}^{-3}$ but it is already $V_{w i}=1.0 \mathrm{~V}$ when $N=2 \times 10^{16} \mathrm{~cm}^{-3}$.

Later on, in addition to the information regarding the doping density and the junction built-in, by changing the position of the quasi-Fermi levels it is possible to obtain information from trap levels within the band-gap, typically characterized by a trap energy $E_{t}$ (with respect to the conduction or valence band levels $E_{C, V}$ for electrons and holes, respectively), capture cross section $\sigma$ and trap density $N_{t}$. Note that $N_{t}$ may respond to deeper defects with lower concentrations than those related with $N$. By modifying the applied bias it varies the relative distance between the hole quasi-Fermi level $E_{F p}$ and $E_{V}$ due to the band bending in the space charge region. Thus the concentration of trapped holes $p_{t}(t)$ in absence of excitation may evolve from $N_{t}$ when the hole traps are emptied in a characteristic time $t=\omega_{e m}{ }^{-1}$, being the emission rate

$$
\omega_{e m}=N_{V} v_{t h} \sigma \exp \left[-E_{t} / k_{B} T\right]
$$


Here, assuming no degeneracy, $N_{V}$ is the effective density of states in the valence band, $v_{t h}$ is the holes thermal velocity, $T$ is the temperature and the product $\omega_{0}=N_{V} v_{t h} \sigma$ is the so called attempt-to-escape frequency (see it arrow-pointed in Figure 2a for the spectrum at $T=300 \mathrm{~K}$ ). The changes in occupation of the trap levels are very $T$-sensitive, modifying $w$ and hence $C_{d l}$, which can be also modified due to the quasi-static charge equilibration in the space charge region. Thus the capacitance behavior under given perturbation conditions informs on $E_{t}, N_{t}$ and $\sigma$. In this sense two of the most general approaches include the use of (i) sinusoidal small perturbations in impedance spectroscopy (IS) analyses and (ii) squared bias pulses for evaluation of time transients.

Within the IS approach, the TAS aims at exploring the density-of-states (DOS) $g(E)$ corresponding to defect levels through the shift of the so-called demarcation $\operatorname{energy}^{16}$

$$
E_{\omega}=k_{B} T \ln \left[\omega_{0} / \omega\right]
$$

where $\omega$ is the angular frequency of the electrical stimulus. In the quasi-steadystate, one defect level of energy $E_{t}$ above $E_{V}$ is occupied as the Fermi level $E_{F}$ crosses it in a given location of the semiconductor bulk. For faster measuring frequencies ( $\left.\omega>\omega_{0}\right)$ or lower temperatures, the defect state is unable to follow the signal as $E_{\omega}$ situates far from the defect level. A contribution to the capacitance cumulatively occurs below $\omega_{0}$, which defines the maximum rate of de-trapping cycles, ${ }^{17}$ when $\omega \approx \omega_{e m}$ ( $\left.E_{\omega} \approx E_{t}\right)$ at that temperature. For slower frequencies $\left(\omega<\omega_{e m}<\omega_{0}\right)$ or higher temperatures, the steady state has been achieved so as to keep the defect occupancy change in-phase with the $a c$ modulation, and thus without energy loss. Accordingly, the 
admittance technique can be viewed as a true energy spectroscopy, producing a traprelated step $\Delta C$ in the capacitance spectrum, as apparently occurs in Figure 2a. Electronic DOS can be easily determined from the capacitance spectrum $C(\omega)$ derivative according to ${ }^{18}$

$$
g\left(E_{\omega}\right)=-\frac{V_{b i} \omega}{q L k_{B} T} \frac{d C(\omega)}{d \omega}
$$

Equation (4) assumes homogeneous trap distribution within the semiconductor bulk and takes $L$ as the absorber layer thickness. Importantly for perovskite solar cells, near full depletion is often attained at zero-bias because of the relatively low doping levels or almost intrinsic character, ${ }^{15,19}$ thus $w \geq L$ and there would be a limited or null validity of Equation (4), and even Equation (1). Furthermore, different defect distributions and band profiles have been discussed in original analyses. ${ }^{18}$

The total trap density can be approached by several means and complex formalisms, ${ }^{20-22}$ however by integration in Equation (4) over frequency (energy) one readily infers a proportionality with the excess capacitance $\Delta C$ (corresponding to the trap-related capacitance step respect to $C_{d l}$, or $C_{g}$ in full depletion) as

$$
N_{t}=\int g\left(E_{\omega}\right) d E_{\omega}=\frac{V_{b i}}{q L} \Delta C
$$

Later from the $g\left(E_{\omega}\right)$ maximum (see Figure $2 \mathrm{~b}$ ), $\omega_{e m}$ are extracted at each $T$ for calculating $E_{t}$ and $\sigma$ in an Arrhenius plot (see inset of Figure 2c), taken as $T$ independent parameters. Differently, the relation $N_{V} v_{t h} \propto T^{2}$ should be considered in Equation (2).

The Arrhenius analysis of Equation (2) is also used in the DLTS technique ${ }^{23}$ but differently to TAS, here the sample is bias pulsed and the resulting capacitance 
transients at each $T$ are measured at a fixed frequency, typically above MHz-range. In absence of traps the capacitance signal may follow the voltage pulse shape. However, assuming the voltage perturbation is actually changing the occupation of the trap level, the capacitance does not return to the $C_{d l}$ value after the pulse. Instead, a modified capacitance $\Delta C$ decays in a time around $\omega_{e m}{ }^{-1}$ at the given $T$. As illustrated in Figure 4a, the transient is sampled twice and the rate window gives $\omega_{e m}$, typically after the identity $t_{2}=t_{1} \exp \left[\omega_{e m}\left(t_{2}-t_{1}\right)\right]$. The sampled capacitance difference $\Delta C_{\omega}(T)=C\left(T, t_{2}\right)-C\left(T, t_{1}\right)$ for each $\omega_{e m}$ peaks at a corresponding $T$ creating the spectroscopic representation. Moreover, the trap density is given as ${ }^{20-23}$

$$
N_{t}=\gamma \frac{N}{C_{d l}} \Delta C
$$

where $\gamma \geq 2$ depending on the measurement conditions (e.g. bias). Note that despite the different nature of perturbations, for reverse bias it can be found that $\gamma \cdot w \rightarrow L$, and thus Equation (6) may approach Equation (5). ${ }^{20-22}$ Accordingly, summing up (i) MS analysis extracts $N$ and $V_{b i}$ from the linear trend $C_{d l}{ }^{-2}(V)$; and subsequently $E_{t}, \sigma$ and $N_{t}$ can be obtained sweeping temperatures: (ii) TAS looks for steps in the $C(\omega)$ spectra in excess of $C_{d l}$ via IS, and (iii) the DLTS evaluates slow $C(t)$ transient changes over $C_{d l}$ after bias step pulses.

In the case of perovskite-based devices, capacitive responses are formed by the contribution of different mechanisms. In addition to geometrical $C_{g}=\varepsilon \varepsilon_{0} / L$ and $C_{d l}$, PSCs show an excess capacitance $C_{s}$ in the low-frequency part of the frequency spectrum ( $f<1 \mathrm{~Hz}$, see Figure 2a). Even in the dark, $C_{s}$ attains values as high as 50 
$\mu \mathrm{F} \mathrm{cm}{ }^{-2}$ measured at $100 \mathrm{mHz}$, much larger than $C_{g}$ typically in the order of $50 \mathrm{nF} \mathrm{cm}{ }^{-2}$ ( $L \approx 400 \mathrm{~nm}, \varepsilon \approx 28)^{24}$ towards high frequencies. The accumulation of mobile ions near the electrode contact has been proposed as suitable explanation through the formation of structures of double-layer kind. ${ }^{25}$ It is also observed that $C_{s}$ is enhanced several orders of magnitude by effect of the incoming light, which illustrate the very strong connection between electronic-ionic processes. ${ }^{26-28}$ Importantly, while the presence of shunt artifacts at very low frequencies probably affects $C_{s}$, the presumably ionic character of this process has been widely tackled with several optoelectronic techniques in relation with the so-called hysteresis of the current-voltage curves in PSCs. ${ }^{15,29}$ Indeed, $C_{s}$ lies behind of capacitive transient currents that affect steady-state operation, thus complicating solar cell characterization and reliability. ${ }^{30}$ Also stability issues have been connected to the occurrence of ionic accumulation/reactivity at the outer interfaces, which are usually visible through featured capacitive responses at low-frequencies. ${ }^{31}$ The experimental distinction among different capacitive mechanisms in such a way that $C_{s}>C_{d l}>C_{g}$ is then necessary in order to thoroughly apply the MS approach. ${ }^{12}$

Mott-Schottky analysis. By examining Figure 1, one can observe two different cases of MS analysis application in PSCs. In the first example (Figure 1a), a dependence of the kind $C^{-2} \propto V$ is distinguishable from quasi-equilibrium to forward bias, which obeys Equation (1) and allows extracting $V_{b i}$ from the intercept of the linear response and $N$ from the slope. At reverse bias full depletion makes the capacitance collapse to $C_{g}$ and at higher forward bias the exponential $C_{s}$ dominates. Note that the linear behavior occurring at least from zero bias towards forward bias is a necessary but not sufficient condition to validate the MS plot. ${ }^{12,15,32}$ There are not too many reports 
showing evident $C_{d l}$ behavior from appropriate MS analysis in PSCs, ${ }^{33-34}$ and Figure 1a corresponding to ref. ${ }^{12}$ is illustrative of the typical comportment of devices with $\mathrm{CH}_{3} \mathrm{NH}_{3} \mathrm{PbI}_{3-x} \mathrm{Cl}_{x}$ as absorber. The calculated defect density resulted $N \approx 10^{17} \mathrm{~cm}^{-3}$, which in fact practically establishes the limit of applicability of the MS technique in PSCs taking into account the latest theoretical simulations suggesting mobile-ions concentrations $N_{s}$ even orders above that. ${ }^{35-36}$ Therefore, the extraction of much lower defect densities becomes masked by additional capacitive mechanisms. ${ }^{12,15}$
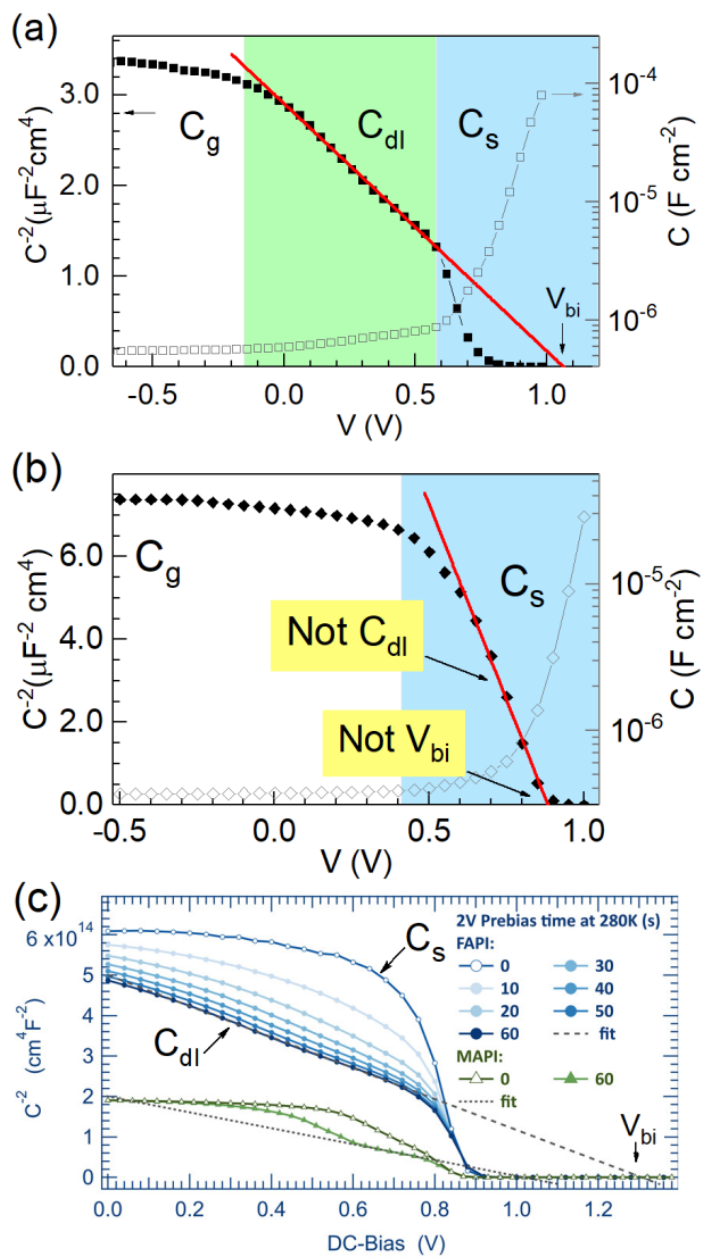

Figure 1. Capacitance-voltage and MS plots of PSCs comprising (a) $\mathrm{CH}_{3} \mathrm{NH}_{3} \mathrm{PbI}_{3-x} \mathrm{Cl}_{x}$ and (b) $\mathrm{CH}_{3} \mathrm{NH}_{3} \mathrm{PbI}_{3}$ illustrating different capacitive regimens. Adapted from ref. ${ }^{12}$ with permission from American Institute of Physics. (c) MS plots for (4) Formamidinium- and 
$\mathrm{CH}_{3} \mathrm{NH}_{3} \mathrm{PbI}_{3}$-based PSCs when different pre-bias durations are applied. Adapted from ref. ${ }^{37}$, Copyright 2018 American Chemical Society.

In the case of Figure $1 \mathrm{~b}$ the MS analysis is unpractical. ${ }^{12}$ Here $N<10^{17} \mathrm{~cm}^{-3}$ and $C_{d l}$ cannot be unambiguously separated from the $C_{s}$ that in fact dominates the capacitive response contribution at typical MS measuring frequencies (1-10 kHz). Furthermore, it has been shown very recently that a transition between these two limiting cases is even observable through MS analysis for formamidinium-based PSCs. ${ }^{37}$ Since mobile defects coexist with fixed impurities, application of pre-bias before capacitance measurement may alter the ionic distribution and the corresponding MS curve, as shown in Figure 1c. However, the effect does not persist and the solar cell relaxes to the initial situation in which the MS analysis is not applicable. ${ }^{12}$

Importantly, from the above arguments every $N$ value extracted from MS analyses in PSCs should be disregarded without the proper checking of the overlapping of $C_{d l}$ with the ion-related $C_{s}$, whose influence gets stronger toward lower frequencies. ${ }^{15}$ However, while it is obviously not encouraged to present bad practices reporting $V_{b i}$ and $N$ from MS plots like Figure $\mathbf{1 b}$ as accurate values in PSCs, ${ }^{38-45}$ what could be useful is to perform qualitative relative comparisons checking changes in the apparent $V_{b i}{ }^{46}$ In this case it should be noticed that the exponential increase of capacitance at forward bias (see Figure 1a,b right axes) is related with the approaching and exceeding of the flat-band potential and the consequent going to the high injection current regime.

Thermal Admittance Spectroscopy. In the TAS approach, excess capacitance has been also recently related to the occupancy of electronic defect states within the band gap of perovskite photovoltaic materials. ${ }^{30,47-54}$ The usual capacitance spectra shape of 
PSCs, as in Figure 2a, present two main features occurring at high and low frequencies, respectively. The plateau at intermediate frequencies $\left(10^{2}-10^{4} \mathrm{~Hz}\right)$ is determined by $C_{g}$, that reduces by effect of the series resistance $R_{\text {series }}$ in the high-frequency part of the spectra. The capacitance increments towards lower frequencies $(0.1-10 \mathrm{~Hz})$, which relates to the ion accumulation effect through $C_{s}$. Since spectra in Figure 2a show two capacitance steps, it is in principle appealing to connect them to the response of defect bands activated at well-separated frequency (energy) ranges.

Accordingly, the capacitance analysis of Equation (4) gives rise to the hypothetical DOS drawn in Figure 2b,c. Two main peaks occur as expected. The highfrequency/low- $E_{\omega}$ signature appears greatly $T$-independent, which more likely responds to the coupling $R_{\text {series }} C_{g}$ that states the dielectric capacitance step (highlighted in Figure 2b), and not to any defect density. Also interfacial layers would have similar influence, ${ }^{55}$ but these are well-known effects in thin film solar cells. ${ }^{2,56-57}$

More interesting is the low-frequency feature. A large, temperature-activated signature is observed attaining huge DOS values $\left(\sim 10^{21} \mathrm{eV}^{-1} \mathrm{~cm}^{-3}\right)$ localized around $E_{\omega} \approx E_{t} \approx 160 \mathrm{meV}$. It should be stressed that these strongly $T$-dependent apparent DOS values are hardly connected to the response of any defect levels. The usual observation of abrupt absorption band tails ${ }^{58-59}$ and the results from Hall effect measurements, ${ }^{30,60}$ suggest that photovoltaic perovskites are slightly doped semiconductors (like in Figure 1b) or at most $N \approx 10^{17}-10^{18} \mathrm{~cm}^{-3}$ (like in Figure 1a).

Disagreeing, capacitance values integrating the low frequency peak after Equation (4) produce total defect densities up to $\sim 10^{20} \mathrm{~cm}^{-3}$ at room temperature, which seems a completely unphysical value. Also the significantly low values extracted for 
$\sigma \approx 10^{-24} \mathrm{~cm}^{2}$ and $\omega_{0}(T=300 \mathrm{~K}) \approx 527 \mathrm{rad} \mathrm{s}^{-1}$ (arrow-pointed in Figure 2a) produce the peculiar representation of measured negative $E_{\omega}$ values in Figure 2c. This is a result of the low frequencies and small activation energy which result in a very low intercept in the Arrhenius plot (Figure 2b), considering that each $E_{t}$ requires his own $E_{\omega}$ axis. ${ }^{2,61}$ In typical semiconductors, traps exhibit dissimilar parameters to those encountered here ( $\sigma>10^{-17} \mathrm{~cm}^{2}$ and $\left.\omega_{0}(T=300 \mathrm{~K})>10^{6} \mathrm{rad} \mathrm{s}^{-1}\right) .^{3,56-57,61}$ Last but not least, we remark that the apparent DOS peaks do not collapse in a unique curve in Figure 2b,c. Instead, a behavior $N_{t} \approx N_{s 0} \exp \left[T / T_{s 0}\right]$ is observed with $N_{s 0} \approx 2 \times 10^{16} \mathrm{~cm}^{-3}$ and $T_{s 0} \approx 34 \mathrm{~K}$ (see inset of Figure 2c), also hardly related to trap states. These parameters, $N_{s 0}$ and $T_{s 0}$, whose physical meanings are still unclear were obtained from the integration of $d C(\omega) / d \ln [\omega]$ and normalized to DOS units as Equation (4). This makes feasible for future works to compare results with other ways of obtaining DOS like thermally stimulated corrents (TSC) and optical techniques as photoluminescence decay. ${ }^{62}$ 

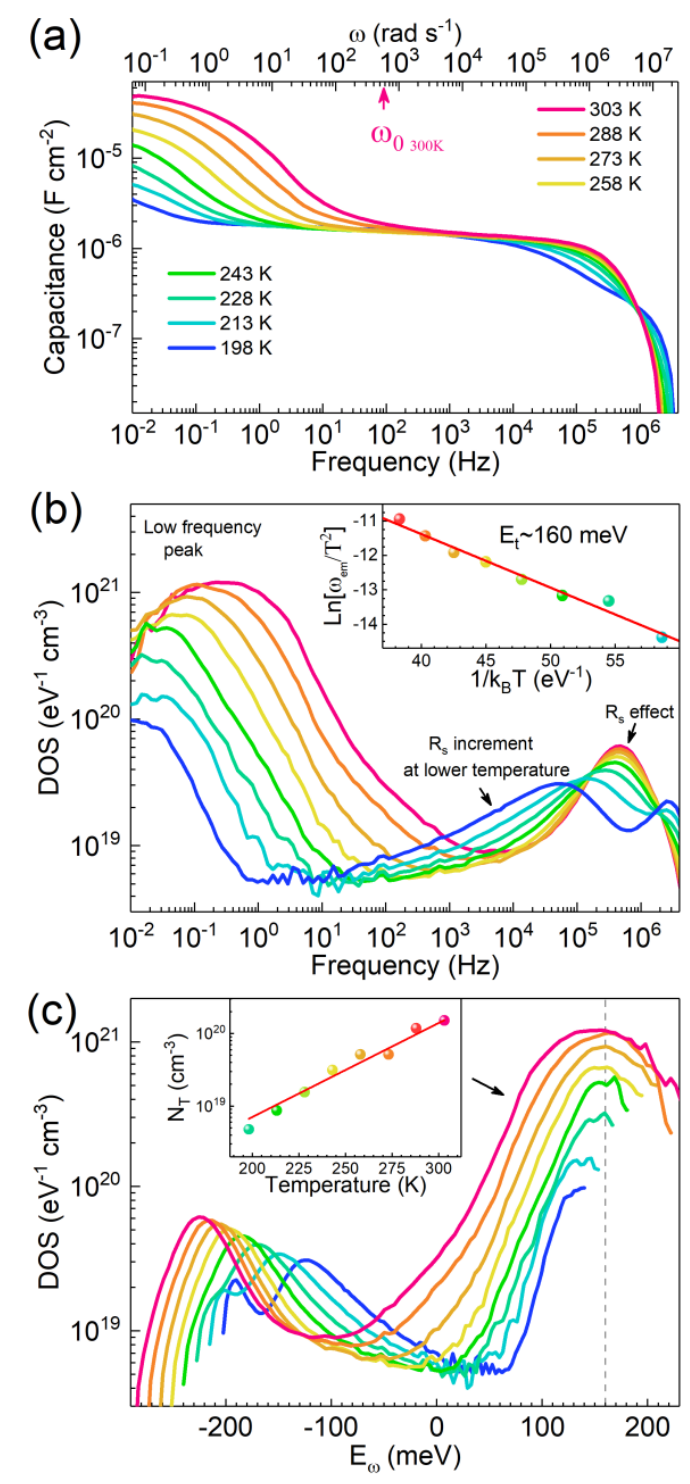

Figure 2. (a) Typical capacitance spectra at varying temperatures and corresponding hypothetical DOS as a function of (b) frequency and (c) demarcation energy of a $\mathrm{CH}_{3} \mathrm{NH}_{3} \mathrm{PbI}_{3}$ ${ }_{\mathrm{x}} \mathrm{Cl}_{\mathrm{x}}$-based PSC, exhibiting two capacitance steps. The DOS is based on Equation (4), assuming $V_{b i}=1.0 \mathrm{~V}$ and $L=200 \mathrm{~nm}$. In inset: (b) the Arrhenius plot extracted from the derivative peak and (c) the trap density dependence on temperature. The $E_{\omega}$ axis in (c) corresponds to the $E_{t}$ from the Arrhenius in (b). Adapted from ref. ${ }^{24}$, Copyright 2015 American Chemical Society.

Hence the interpretation of the low-frequency capacitance step in terms of the 
occupancy of bulk electronic DOS would entail $\Delta C \propto L$ clearly in contradiction with previous observations. Indeed, thickness-independent capacitance observed in the dark is consistent with the electrode polarization at perovskite/contact layer interfaces cause by mobile ions accumulation as widely admitted. ${ }^{24}$ Therefore, we can conclude that, at least of PSCs comprising oxide selective contacts, the large low-frequency capacitance dominates the electrical response, masking possible capacitive contributions produced by electronic transitions. As known, the use of fullerene derivatives as electron extracting layers produces a reduction in the low-frequency capacitance contribution. ${ }^{63}$ By lowering $C_{s}$, its masking effect becomes less pronounced so as to reveal true defect bands within the band-gap. This is suggested by the analysis in Figure $\mathbf{3}$ that compares room-temperature capacitance spectra of PSCs with different structures comprising $\mathrm{TiO}_{2}$ and fullerene layers. While the low-frequency capacitance dominates the response for oxide-based contacts, the solar cell comprising fullerene electron extracting layers exhibits a well-defined peak around $10^{4} \mathrm{~Hz}$, which might be related to bulk defect responses. This signature needs of further exploration to clearly elucidate its origin. 

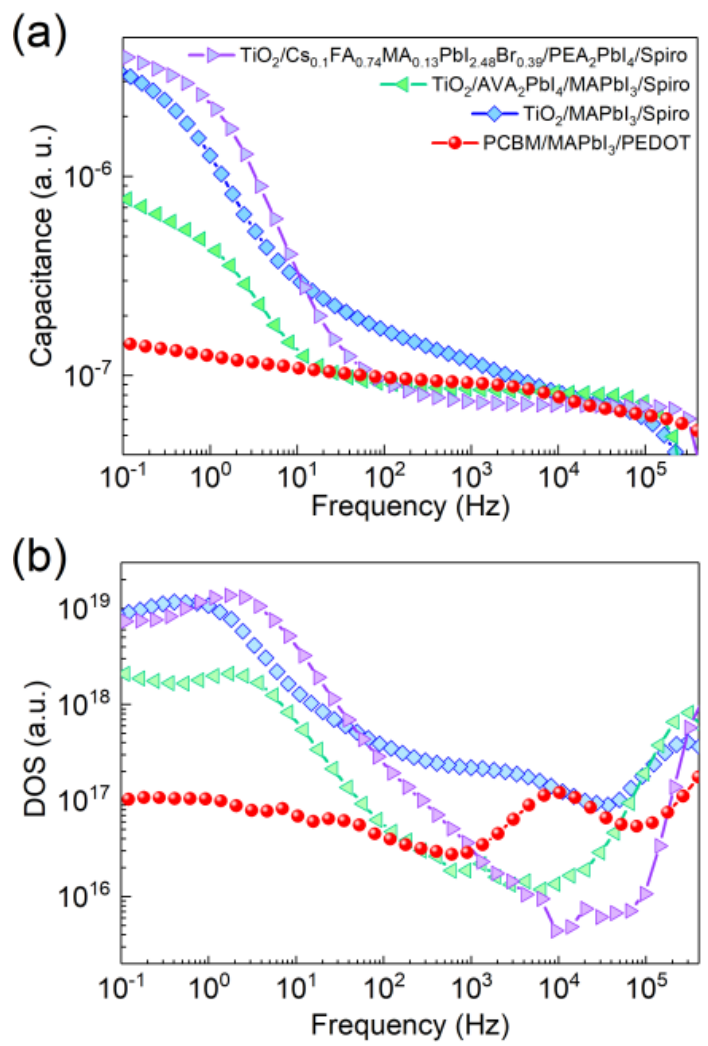

Figure 3. Comparison of (a) capacitance and (b) corresponding DOS spectra at room temperature for different PSCs structures, as indicated. Vertical axes are shifted for better comparison. Data adapted with permission from ref. ${ }^{31}$, Copyright 2016 American Chemical Society, and from ref. ${ }^{28}$, Copyright 2018 Elsevier.

Deep Level Transient Spectroscopy. The use of DLTS as a parameter extracting tool has been also recently reported for PSCs. ${ }^{64-69}$ The general spectra patterns include peaks around and above $300 \mathrm{~K}$ in a broad range of rate windows $(\mathrm{Hz}-\mathrm{kHz})$ (see Figure 4b). Here once again the masking effect of $C_{s}$ excess capacitance should be noticed, as highlighted in the scheme of Figure 4a with thinner red lines. The slow evolving capacitance could generate its own peaks or shift trap-related ones. The actual appearance of these phenomena is illustrated in Figure 4c for samples with symmetric contacts; i.e. without rectifying behavior. There transient capacitance from perovskite 
pellets is showed following typical Gouy-Chapman ionic theory as $C_{s} \approx \varepsilon \varepsilon_{0} L_{D}{ }^{-1} \cosh \left[q V / 2 k_{B} T\right](1-\exp [t / \tau])$ meaning diffuse charge layers of Debye lengths $L_{D} \approx 200 \mathrm{~nm}$ and relaxation times $\tau$ around seconds. ${ }^{25}$

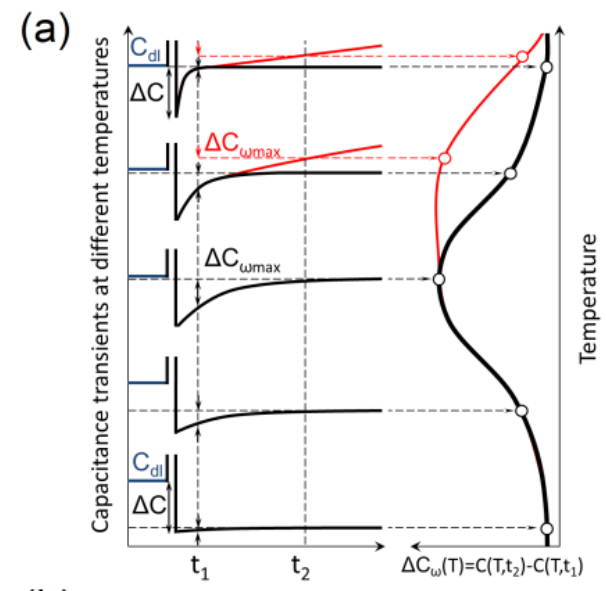

(b)
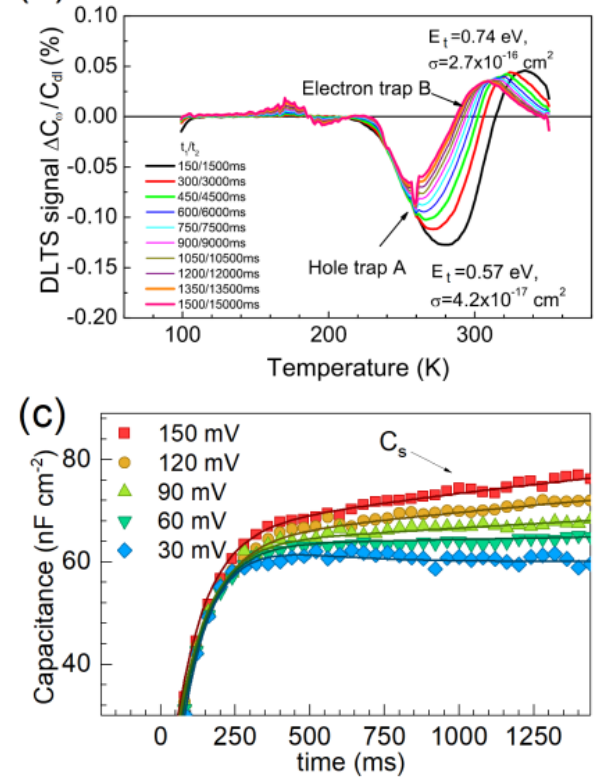

Figure 4. (a) Scheme of the basic working principle of DLTS measurement; in thinner red lines a possible masking effect of slow evolving capacitance transients likely due to ionic related processes. (b) DLTS spectra for a $\mathrm{Cs}_{\mathrm{x}}\left(\mathrm{MA}_{0.17} \mathrm{FA}_{0.83}\right)_{(1-\mathrm{x})} \mathrm{Pb}\left(\mathrm{I}_{0.83} \mathrm{Br}_{0.17}\right)_{3}$ perovskite incorporated in mesoscopic solar cells. (c) Capacitance transients from $\mathrm{CH}_{3} \mathrm{NH}_{3} \mathrm{PbI}_{3}$ pellets for different voltage pulse amplitudes. Adapted from references ${ }^{64}$ and ${ }^{25}$, respectively, with permission from American Institute of Physics. 
Schematizing and summing up our discussion, the left panel of Figure 5 displays the well-known energy diagram (a), the corresponding charge density profile (b), and charge variation under perturbation across depth (c) for a deep trap level in a $p$-type semiconductor towards the junction (cathode). There the bands bend in the depletion region and there is an extra step in the charge density profile at the position $x_{t}$ where $E_{F}$ crosses the trap level and changes its electronic occupancy. In Figure 5b left panel, the $N$ and $N_{t}$ correspond to homogeneous ionized shallow doping and trap level contributions, respectively. Accordingly, the space charge varies around $w$ and $x_{t}$ when a perturbation is applied (Figure $5 \mathrm{c}$ left panel), giving rise to the measured $C_{d l}$ and $\Delta C$ . However, PSCs seem to be not accurately described by the left panel of Figure 5, since mobile ions generate a more complex profile altering the band bending and/or broad shallower/deeper gap states. Keeping the patterns of the left panel with thinner lines for comparison in the right side of Figure 5, a hypothetical example of mobile ion distribution is shown with thicker red lines. Note that for simplicity we only illustrate mobile anions (“-” red circles in Figure 5a right panel) in a typical exponential-like distribution towards the cathode, resembling the Gouy-Chapman diffuse layer distribution caused by electrode polarization. ${ }^{25}$ As a result, modifications $\Delta w$ in the depletion width are expected (Figure 5a right panel), sometimes even achieving full depletion, with the consequent increase of mobile cations and/or ionized donors, e.g. at the cathode or towards the anode, respectively. Furthermore, the steps in the charge density profile can be shifted, as in Figure 5b right panel, and the constant profile approximation may hold hardly. Thus, time perturbations across the depth axis could sense charge changes everywhere within the absorber, peaking at $x_{s 1}$ and $x_{s 2}$, and even 
more important towards extra positions like the cathode interface $x_{s 0}$, overlapping charge contributions as in Figure 5c right panel. Note that the $N_{s}(x, t)$ profiles should not be taken as spatial simple exponential/linear distributions to be added to $N_{t}+N$.

Instead, a more dynamic time-evolving phenomena should be considered where a broad range of distributions may occur, as in the orange squares of Figure 5c. This points out the non-static character of $N_{s}$, which modifies the capacitance spectra and originates the hysteresis phenomena. Therefore, $C_{s}$-related charge overlaps both $\Delta C$ and $C_{d l}$ around $x_{t}$ and $w$, respectively, and produces its complete masking.

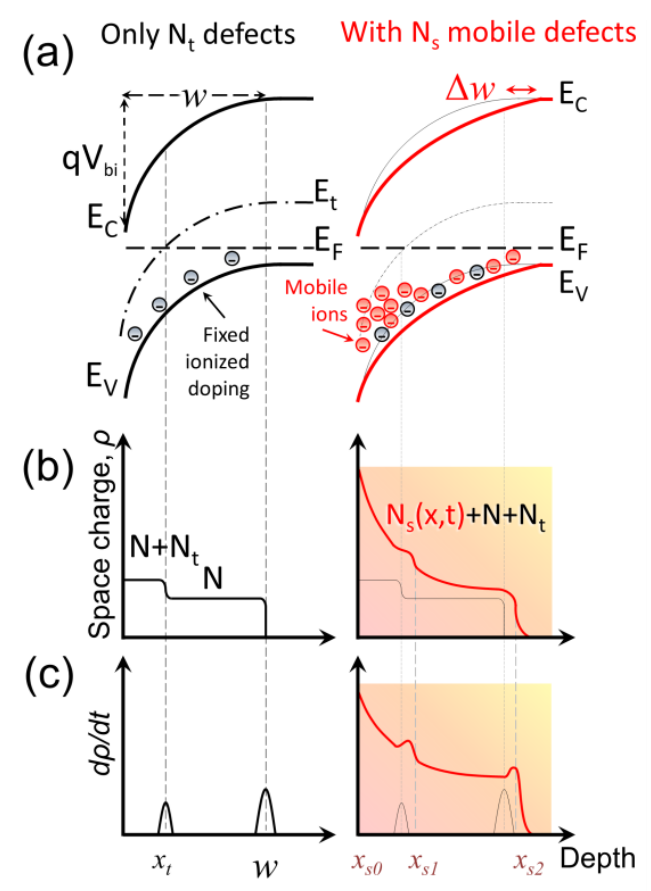

Figure 5. (a) Schematic band energy diagram, (b) charge density profile and (c) absolute charge variation upon perturbation, e.g. $d V / d t$, of a $p$-type absorber perovskite towards the $n$ type contact (left) including a deep trap state neglecting (left panel) and including (right panel) mobile ionic effects, respectively. The patterns of left panel are reproduced with thinner lines in the right panels for comparison purposes. The orange square signals the broad region where 
the patterns of $N_{s}(x, t)$ could lie, despite in the example only anions are displayed with an exponential-like distribution towards the cathode. For (b) and (c) approximate semi-log scaling should be understood.

From the above considerations, we highlight the lack of systematic studies accounting for and describing the role of the ionic-like capacitances and its masking effect on techniques like TAS and DLTS. Here it is advisable to check the consistency of the spectra with geometrical changes like the bulk thickness or the material properties itself, where expected trap density modifications may occur. This is crucial for clarifying the role of deep trap states in $C_{s}$ or its nature/localization in self. Interestingly, and particularly regarding the lower values of $\sigma$ from TAS, it is also still lacking a study checking the Meyer-Neldel rule in PSCs; i.e. $\sigma=\sigma_{0} \exp \left[E_{t} / k_{B} T_{t}\right]$ in Equation (2). ${ }^{1,70-71}$ If satisfied the latter rule, it would suggest that the $C_{s}$-related $E_{t}$ is the enthalpy increase $\Delta H$ for the thermodynamic system upon ionization at a given $T$, with the corresponding entropy change as $\Delta S=E_{t} / T_{t}$.

Also the combination of bias perturbation with optical variants with the earlier considerations may provide better understanding. Particularly in relation with the TAS, it has been recently reported that the low frequency region of the spectra from intensitymodulating photocurrent spectroscopy (IMPS, ac light perturbation $\tilde{\Phi}$ for photocurrent signal $\tilde{J}_{\Phi}$ ) in PSCs deliver extra capacitance contributions not discernible from IS. ${ }^{72}$ In this sense the next step would be to complement the IMPS with intensity-modulated photo-voltage spectroscopy (IMVS, ac light perturbation $\tilde{\Phi}$ for photo-voltage signal $\tilde{V}_{\Phi}$ ) in such a way that the impedance $Z_{\Phi}(\omega)=\left(\tilde{V}_{\Phi} / \tilde{\Phi}\right) /\left(\tilde{J}_{\Phi} / \tilde{\Phi}\right)=\tilde{V}_{\Phi} / \tilde{J}_{\Phi}$ can be 
defined. ${ }^{73}$ From the latter, a light-modulated thermal admittance spectroscopy (LMTAS) could be implemented by applying Equation (4) to the $\operatorname{Re}\left[C_{\Phi}(\omega)=-i / \omega Z_{\Phi}(\omega)\right]$ in PSCs.

In summary, the application of the MS analysis of the depletion layer capacitance in PSCs suffers a masking effect caused by interfacial and ion-related excess capacitances $\left(C_{d l}+C_{s}\right)$. Although $C_{s}$ dominates the low-frequency response, it spreads to the mid/high-frequency range and overlaps additional electronically-caused defect signals. This misleads the MS analysis and creates "fake" or overlapping peaks in the spectra from TAS and DLTS measurements $\left(\Delta C+C_{s}\right)$ when characterizing deeper defect levels. These problematic may require a series of systematic studies in order to make reliable deep trap level characterizations in PSCs, particularly for those devices that exhibit less pronounced excess capacitances. In this sense, it would be strategic to check variations in the device geometry and material architecture, as well as to combine characterizations with bias and light perturbations.

\section{AUTHOR INFORMATION}

\section{Corresponding Authors}

*E-mail: osbel.almora@ fau.de; garciag@uji.es

\section{ORCID}

Osbel Almora: $\underline{0000-0002-2523-0203}$

Marisé García-Batlle: 0000-0002-9142-2430

Germà Garcia-Belmonte: $\underline{0000-0002-0172-6175}$

\section{Notes}

The authors declare no competing financial interest. 


\section{Biographies}

Osbel Almora graduated in Physics from the University of Havana, Cuba, in 2013 and, since 2017, he is been a PhD candidate in the chair of Prof. Christoph J. Brabec at the Friedrich-Alexander-Universität Erlangen-Nürnberg, Germany, also co-supervised by Prof. Germà Garcia-Belmonte from the Universitat Jaume I de Castelló, Spain. Osbel's main topic of interest is the characterization and modeling of energy devices.

Marisé García-Batlle (BSc. in Radiochemistry, INSTEC Havana, Cuba 2014 and MSc. in Chemistry Sciences, UNAM, Mexico, 2017) is currently working as a pre-doctoral candidate at the Institute of Advanced Materials in the Universitat Jaume I of Castelló, Spain, supervised by Prof. Germà Garcia-Belmonte in the field of photovoltaics and optoelectronics devices.

Germà Garcia-Belmonte joined the Universitat Jaume I of Castelló in 1992 and currently works as a Full Professor of Applied Physics (2010) at the Institute of Advanced Materials (http://www.inam.uji.es/ ). He conducts experimental and theoretical research on materials and devices for production and storage of clean energies, in particular, on electronics and ionics of energy devices. He is currently active in the topic of perovskite-based solar cells and lithium-ion batteries. Device physics using impedance spectroscopy is his main expertise. He is listed as 2018 Highly Cited Researcher (Clarivate Analytics) in the cross-field category.

\section{ACKNOWLEDGMENTS}

We acknowledge funding from MINECO of Spain under Projects MAT201676892-C3-1-R. O.A. acknowledges the VDI/VD Innovation + Technik GmbH (Projecttitle: PV-ZUM) and the SAOT funded by the German Research Foundation (DFG) in the framework of the German excellence initiative. M. G.-B. also acknowledges 
Generalitat Valenciana for the grant GRISOLIAP/2018/073.

\section{REFERENCES}

(1) Heath, J.; Zabierowski, P. Capacitance Spectroscopy of Thin-Film Solar Cells. In Advanced Characterization Techniques for Thin Film Solar Cells, Abou-Ras, D.; Kirchartz, T.; Rau, U., Eds. Wiley-VCH Verlag GmbH \& Co. KGaA: Boschstr. 12, 69469 Weinheim, Germany, 2011, pp 81-106.

(2) Li, J. V.; Ferrari, G. Capacitance Spectroscopy of Semiconductors. 1st ed.; Taylor \& Francis Group: New York, 2018, p 460.

(3) Wang, S.; Kaienburg, P.; Klingebiel, B.; Schillings, D.; Kirchartz, T. Understanding Thermal Admittance Spectroscopy in Low-Mobility Semiconductors. $J$. Phys. Chem. C 2018, 122, 9795-9803.

(4) Li, B.; Ferguson, V.; Silva, S. R. P.; Zhang, W. Defect Engineering toward Highly Efficient and Stable Perovskite Solar Cells. Adv. Mater. Interfaces 2018, 5, 1800326.

(5) Ran, C.; Xu, J.; Gao, W.; Huang, C.; Dou, S. Defects in Metal Triiodide Perovskite Materials towards High-Performance Solar Cells: Origin, Impact, Characterization, and Engineering. Chem. Soc. Rev. 2018, 47, 4581-4610.

(6) Wu, H.; Li, D.; Shi, J.; Luo, Y.; Xu, Y.; Meng, Q. Inorganic-Organic Halide Perovskites for New Photovoltaic Technology. Natl. Sci. Rev. 2017, 5, 559-576.

(7) Wang, F.; Bai, S.; Tress, W.; Hagfeldt, A.; Gao, F. Defects Engineering for High-Performance Perovskite Solar Cells. npj Flexible Electronics 2018, 2, 22.

(8) Correa-Baena, J.-P.; Abate, A.; Saliba, M.; Tress, W.; Jesper Jacobsson, T.; Grätzel, M.; Hagfeldt, A. The Rapid Evolution of Highly Efficient Perovskite Solar Cells. Energy Environm. Sci. 2017, 10, 710-727.

(9) Kim, D. H.; Whitaker, J. B.; Li, Z.; van Hest, M. F. A. M.; Zhu, K. Outlook and Challenges of Perovskite Solar Cells toward Terawatt-Scale Photovoltaic Module Technology. Joule 2018, 2, 1437-1451.

(10) Zarazua, I.; Han, G.; Boix, P. P.; Mhaisalkar, S.; Fabregat-Santiago, F.; Mora-Seró, I.; Bisquert, J.; Garcia-Belmonte, G. Surface Recombination and Collection Efficiency in Perovskite Solar Cells from Impedance Analysis. J. Phys. Chem. Lett. 2016, 7, 5105-5113. 
(11) Chen, J.; Park, N.-G. Causes and Solutions of Recombination in Perovskite Solar Cells. Adv. Mater. 2019, DOI: 10.1002/adma.201803019.

(12) Almora, O.; Aranda, C.; Mas-Marzá, E.; Garcia-Belmonte, G. On MottSchottky Analysis Interpretation of Capacitance Measurements in Organometal Perovskite Solar Cells. Appl. Phys. Lett. 2016, 109, 173903.

(13) Schottky, W. Vereinfachte und erweiterte Theorie der Randschichtgleichrichter. Z. Phys. 1942, 118, 539-592.

(14) van Opdorp, C. Evaluation of Doping Profiles from Capacitance Measurements. Solid-State Electron. 1968, 11, 397-406.

(15) Lopez-Varo, P.; Jiménez-Tejada, J. A.; García-Rosell, M.; Ravishankar, S.; Garcia-Belmonte, G.; Bisquert, J.; Almora, O. Device Physics of Hybrid Perovskite Solar Cells: Theory and Experiment. Adv. Energy Mater. 2018, 8, 1702772.

(16) Hegedus, S. S.; Fagen, E. A. Midgap States in a-Si:H and a-SiGe:H p-i-n Solar Cells and Schottky Junctions by Capacitance Techniques. J. Appl. Phys. 1992, 71, 5941-5951.

(17) Carr, J. A.; Elshobaki, M.; Chaudhary, S. Deep Defects and the Attempt to Escape Frequency in Organic Photovoltaic Materials. Appl. Phys. Lett. 2015, 107, 203302.

(18) Walter, T.; Herberholz, R.; Müller, C.; Schock, H. W. Determination of Defect Distributions from Admittance Measurements and Application to $\mathrm{Cu}(\mathrm{In}, \mathrm{Ga}) \mathrm{Se}_{2}$ Based Heterojunctions. J. Appl. Phys. 1996, 80, 4411-4420.

(19) Guerrero, A.; Juarez-Perez, E. J.; Bisquert, J.; Mora-Sero, I.; GarciaBelmonte, G. Electrical Field Profile and Doping in Planar Lead Halide Perovskite Solar Cells. App. Phys. Lett. 2014, 105, 133902.

(20) Mooney, P. M. Defect Identification Using Capacitance Spectroscopy. In Semiconductors and Semimetals, Stavola, M., Ed. Elsevier: 1999, pp 93-152.

(21) Blood, P.; Orton, J. W. The Electrical Characterization of Semiconductors: Majority Carriers and Electron States. Academic Press: Oxford, England, 1992; p 735.

(22) Auth, J. On the Reverse-Biased Capacitance of Step p+-n Junctions with Traps. Phys. Status Solidi B 1968, 27, 653-656.

(23) Miller, G. L.; Lang, D. V.; Kimerling, L. C. Capacitance Transient 
Spectroscopy. Annu. Rev. Mater. Sci. 1977, 7, 377-448.

Almora, O.; Zarazua, I.; Mas-Marza, E.; Mora-Sero, I.; Bisquert, J.;

Garcia-Belmonte, G. Capacitive Dark Currents, Hysteresis, and Electrode Polarization in Lead Halide Perovskite Solar Cells. J. Phys. Chem. Lett. 2015, 6, 1645-1652.

(25) Almora, O.; Guerrero, A.; Garcia-Belmonte, G. Ionic Charging by Local Imbalance at Interfaces in Hybrid Lead Halide Perovskites. Appl. Phys. Lett. 2016, 108, 043903.

(26) Juarez-Perez, E. J.; Sanchez, R. S.; Badia, L.; Garcia-Belmonte, G.; Kang, Y. S.; Mora-Sero, I.; Bisquert, J. Photoinduced Giant Dielectric Constant in Lead Halide Perovskite Solar Cells. J. Phys. Chem. Lett. 2014, 5, 2390-2394.

(27) Almora, O.; Aranda, C.; Garcia-Belmonte, G. Do Capacitance Measurements Reveal Light-Induced Bulk Dielectric Changes in Photovoltaic Perovskites? J. Phys. Chem. C 2018, 122, 13450-13454.

(28) Almora, O.; Cho, K. T.; Aghazada, S.; Zimmermann, I.; Matt, G. J.; Brabec, C. J.; Nazeeruddin, M. K.; Garcia-Belmonte, G. Discerning Recombination Mechanisms and Ideality Factors through Impedance Analysis of High-Efficiency Perovskite Solar Cells. Nano Energy 2018, 48, 63-72.

(29) Snaith, H. J.; Abate, A.; Ball, J. M.; Eperon, G. E.; Leijtens, T.; Noel, N. K.; Stranks, S. D.; Wang, J. T.-W.; Wojciechowski, K.; Zhang, W. Anomalous Hysteresis in Perovskite Solar Cells. J. Phys. Chem. Lett. 2014, 5, 1511-1515.

(30) Shao, Y.; Xiao, Z.; Bi, C.; Yuan, Y.; Huang, J. Origin and Elimination of Photocurrent Hysteresis by Fullerene Passivation in $\mathrm{CH}_{3} \mathrm{NH}_{3} \mathrm{PbI}_{3}$ Planar Heterojunction Solar Cells. Nat. Commun. 2014, 5, 5784.

(31) Almora, O.; Aranda, C.; Zarazua, I.; Guerrero, A.; Garcia-Belmonte, G. Noncapacitive Hysteresis in Perovskite Solar Cells at Room Temperature. ACS Energy Lett. 2016, 1, 209-215.

(32) Werner, F.; Babbe, F.; Burkhart, J.; Spindler, C.; Elanzeery, H.; Siebentritt, S. Interdiffusion and Doping Gradients at the Buffer/Absorber Interface in Thin-Film Solar Cells. ACS Appl. Mater. Interfaces 2018, 10, 28553-28565.

(33) Guerrero, A.; Garcia-Belmonte, G.; Mora-Sero, I.; Bisquert, J.; Kang, Y. S.; Jacobsson, T. J.; Correa-Baena, J.-P.; Hagfeldt, A. Properties of Contact and Bulk Impedances in Hybrid Lead Halide Perovskite Solar Cells Including Inductive Loop 
Elements. J. Phys. Chem. C 2016, 120, 8023-8032.

(34) Mahmud, M. A.; Elumalai, N. K.; Upama, M. B.; Wang, D.; Chan, K. H.; Wright, M.; Xu, C.; Haque, F.; Uddin, A. Low Temperature Processed ZnO Thin Film as Electron Transport Layer for Efficient Perovskite Solar Cells. Sol. Energy Mater. Sol. Cells 2017, 159, 251-264.

(35) Almora, O.; Lopez-Varo, P.; Cho, K. T.; Aghazada, S.; Meng, W.; Hou, Y.; Echeverría-Arrondo, C.; Zimmermann, I.; Matt, G. J.; Jiménez-Tejada, J. A.; Brabec, C. J.; Nazeeruddin, M. K.; Garcia-Belmonte, G. Ionic Dipolar Switching Hinders Charge Collection in Perovskite Solar Cells with Normal and Inverted Hysteresis. Sol. Energy Mater. Sol. Cells 2019, 195, 291-298.

(36) Moia, D.; Gelmetti, I.; Calado, P.; Fisher, W.; Stringer, M.; Game, O.; Hu, Y.; Docampo, P.; Lidzey, D.; Palomares, E.; Nelson, J.; Barnes, P. R. F. Ionic-toElectronic Current Amplification in Hybrid Perovskite Solar Cells: Ionically Gated Transistor-Interface Circuit Model Explains Hysteresis and Impedance of Mixed Conducting Devices. Energy Environm. Sci. 2019, 12, 1296-1308.

(37) Fischer, M.; Tvingstedt, K.; Baumann, A.; Dyakonov, V. Doping Profile in Planar Hybrid Perovskite Solar Cells Identifying Mobile Ions. ACS Appl. Energy Mater. 2018, 1, 5129-5134.

(38) Wei, W.; Hu, B.; Jin, F.; Jing, Z.; Li, Y.; García Blanco, A. A.; Stacchiola, D. J.; Hu, Y. H. Potassium-Chemical Synthesis of 3D Graphene from $\mathrm{CO}_{2}$ and its Excellent Performance in HTM-free Perovskite Solar Cells. J. Mater. Chem. A 2017, 5, 7749-7752.

(39) Huang, Y.; Aharon, S.; Rolland, A.; Pedesseau, L.; Durand, O.; Etgar, L.; Even, J. Influence of Schottky Contact on the C-V and J-V Characteristics of HTM-free Perovskite Solar Cells. EPJ Photovolt. 2017, 8, 85501.

(40) Brus, V. V.; Lang, F.; Bundesmann, J.; Seidel, S.; Denker, A.; Rech, B.; Landi, G.; Neitzert, H. C.; Rappich, J.; Nickel, N. H. Defect Dynamics in Proton Irradiated $\mathrm{CH}_{3} \mathrm{NH}_{3} \mathrm{PbI}_{3}$ Perovskite Solar Cells. Adv. Electron. Mater. 2017, 3, 1600438.

(41) Heinrichsdobler, A.; Engelmayer, M.; Riedel, D.; Brabec, C. J.; Wehlus, T. Inkjet-Printed Polymer-Based Scattering Layers for Enhanced Light Outcoupling from Top-Emitting Organic Light-Emitting Diodes. In Organic Light Emitting Materials and Devices XXI, So, F.; Adachi, C.; Kim, J.-J., Eds. SPIE: 2017, p 103622B. 
(42) Khadka, D. B.; Shirai, Y.; Yanagida, M.; Ryan, J. W.; Miyano, K. Exploring the Effects of Interfacial Carrier Transport Layers on Device Performance and Optoelectronic Properties of Planar Perovskite Solar Cells. J. Mater. Chem. C 2017, $5,8819-8827$.

(43) Yang, G.; Wang, C.; Lei, H.; Zheng, X.; Qin, P.; Xiong, L.; Zhao, X.; Yan, Y.; Fang, G. Interface Engineering in Planar Perovskite Solar Cells: Energy Level Alignment, Perovskite Morphology Control and High Performance Achievement. $J$. Mater. Chem. A 2017, 5, 1658-1666.

(44) Chouhan, A. S.; Jasti, N. P.; Avasthi, S. Effect of Interface Defect Density on Performance of Perovskite Solar Cell: Correlation of Simulation and Experiment. Mater. Lett. 2018, 221, 150-153.

(45) Zohar, A.; Kulbak, M.; Levine, I.; Hodes, G.; Kahn, A.; Cahen, D. What Limits the Open-Circuit Voltage of Bromide Perovskite-Based Solar Cells? ACS Energy Lett. 2019, 4, 1-7.

(46) Guerrero, A.; Bou, A.; Almora, O.; Heumuller, T.; Garcia-Belmonte, G.; Bisquert, J.; Hou, Y.; Brabec, C. J. Switching Off Hysteresis in Perovskite Solar Cells by Fine-tuning Energy Levels of Extraction Layers. Adv. Energy Mater. 2018, 8, 201703376.

(47) Duan, H.-S.; Zhou, H.; Chen, Q.; Sun, P.; Luo, S.; Song, T.-B.; Bob, B.; Yang, Y. The Identification and Characterization of Defect States in Hybrid OrganicInorganic Perovskite Photovoltaics. Phys. Chem. Chem. Phys. 2015, 17, 112-116.

(48) Xiao, Z.; Dong, Q.; Bi, C.; Shao, Y.; Yuan, Y.; Huang, J. Solvent Annealing of Perovskite-Induced Crystal Growth for Photovoltaic-Device Efficiency Enhancement. Adv. Mater. 2014, 26, 6503-6509.

(49) Samiee, M.; Konduri, S.; Ganapathy, B.; Kottokkaran, R.; Abbas, H. A.; Kitahara, A.; Joshi, P.; Zhang, L.; Noack, M.; Dalal, V. Defect Density and Dielectric Constant in Perovskite Solar Cells. Appl. Phys. Lett. 2014, 105, 153502.

(50) Lee, J.-W.; Kim, D.-H.; Kim, H.-S.; Seo, S.-W.; Cho, S. M.; Park, N.-G. Formamidinium and Cesium Hybridization for Photo- and Moisture-Stable Perovskite Solar Cell. Adv. Energy Mater. 2015, 5, 1501310.

(51) Lin, Y.; Chen, B.; Zhao, F.; Zheng, X.; Deng, Y.; Shao, Y.; Fang, Y.; Bai, Y.; Wang, C.; Huang, J. Matching Charge Extraction Contact for Wide-Bandgap 
Perovskite Solar Cells. Adv. Mater. 2017, 29, 1700607.

(52) Lin, Y.; Shen, L.; Dai, J.; Deng, Y.; Wu, Y.; Bai, Y.; Zheng, X.; Wang, J.; Fang, Y.; Wei, H.; Ma, W.; Zeng, X. C.; Zhan, X.; Huang, J. $\pi$-Conjugated Lewis Base: Efficient Trap-Passivation and Charge-Extraction for Hybrid Perovskite Solar Cells. Adv. Mater. 2017, 29, 1604545.

(53) Wei, W.; Zhang, Y.; Xu, Q.; Wei, H.; Fang, Y.; Wang, Q.; Deng, Y.; Li, T.; Gruverman, A.; Cao, L.; Huang, J. Monolithic Integration of Hybrid Perovskite Single Crystals with Heterogenous Substrate for Highly Sensitive X-Ray Imaging. Nat. Photon. 2017, 11, 315.

(54) Tan, H.; Che, F.; Wei, M.; Zhao, Y.; Saidaminov, M. I.; Todorović, P.; Broberg, D.; Walters, G.; Tan, F.; Zhuang, T.; Sun, B.; Liang, Z.; Yuan, H.; Fron, E.; Kim, J.; Yang, Z.; Voznyy, O.; Asta, M.; Sargent, E. H. Dipolar Cations Confer Defect Tolerance in Wide-Bandgap Metal Halide Perovskites. Nat. Commun. 2018, 9, 3100.

(55) Werner, F.; Siebentritt, S. Buffer Layers, Defects, and the Capacitance Step in the Admittance Spectrum of a Thin-Film Solar Cell. Phys. Rev. Appl. 2018, 9, 054047.

(56) Kneisel, J.; Siemer, K.; Luck, I.; Bräunig, D. Admittance Spectroscopy of Efficient CuInS 2 Thin Film Solar Cells. J. Appl. Phys. 2000, 88, 5474-5481.

(57) Weiss, T. P.; Redinger, A.; Luckas, J.; Mousel, M.; Siebentritt, S. In Role of high Series Resistance in Admittance Spectroscopy of Kesterite Solar Cells, 2013 IEEE 39th Photovoltaic Specialists Conference (PVSC), Tampa, FL, USA, 16-21 June 2013; IEEE: Tampa, FL, USA, 2013; pp 3066-3070.

(58) De Wolf, S.; Holovsky, J.; Moon, S.-J.; Löper, P.; Niesen, B.; Ledinsky, M.; Haug, F.-J.; Yum, J.-H.; Ballif, C. Organometallic Halide Perovskites: Sharp Optical Absorption Edge and Its Relation to Photovoltaic Performance. J. Phys. Chem. Lett. 2014, 5, 1035-1039.

(59) Wright, A. D.; Milot, R. L.; Eperon, G. E.; Snaith, H. J.; Johnston, M. B.; Herz, L. M. Band-Tail Recombination in Hybrid Lead Iodide Perovskite. Adv. Funct. Mater. 2017, 27, 1700860.

(60) Chen, Y.; Yi, H. T.; Wu, X.; Haroldson, R.; Gartstein, Y. N.; Rodionov, Y. I.; Tikhonov, K. S.; Zakhidov, A.; Zhu, X. Y.; Podzorov, V. Extended Carrier Lifetimes and Diffusion in Hybrid Perovskites Revealed by Hall Effect and 
Photoconductivity Measurements. Nat. Commun. 2016, 7, 12253.

(61) Weiss, T. P.; Redinger, A.; Regesch, D.; Mousel, M.; Siebentritt, S.

Direct Evaluation of Defect Distributions From Admittance Spectroscopy. IEEE J. Photovoltaics 2014, 4, 1665-1670.

(62) Tress, W. Perovskite Solar Cells on the Way to Their Radiative Efficiency Limit - Insights Into a Success Story of High Open-Circuit Voltage and Low Recombination. Adv. Energy Mater. 2017, 7, 1602358.

(63) Kim, H.-S.; Jang, I.-H.; Ahn, N.; Choi, M.; Guerrero, A.; Bisquert, J.; Park, N.-G. Control of I-V Hysteresis in $\mathrm{CH}_{3} \mathrm{NH}_{3} \mathrm{PbI}_{3}$ Perovskite Solar Cell. J. Phys. Chem. Lett. 2015, 6, 4633-4639.

(64) Polyakov, A. Y.; Smirnov, N. B.; Shchemerov, I. V.; Saranin, D. S.; Le, T. S.; Didenko, S. I.; Kuznetsov, D. V.; Agresti, A.; Pescetelli, S.; Matteocci, F.; Carlo, A. D. Trap States in Multication Mesoscopic Perovskite Solar Cells: A Deep Levels Transient Spectroscopy Investigation. Appl. Phys. Lett. 2018, 113, 263501.

(65) Heo, S.; Seo, G.; Lee, Y.; Lee, D.; Seol, M.; Lee, J.; Park, J.-B.; Kim, K.; Yun, D.-J.; Kim, Y. S.; Shin, J. K.; Ahn, T. K.; Nazeeruddin, M. K. Deep Level Trapped Defect Analysis in $\mathrm{CH}_{3} \mathrm{NH}_{3} \mathrm{PbI}_{3}$ Perovskite Solar Cells by Deep Level Transient Spectroscopy. Energy Environ. Sci. 2017, 10, 1128-1133.

(66) Hsieh, H.-C.; Hsiow, C.-Y.; Lin, K.-F.; Shih, Y.-C.; Wang, L.; Renaud, C.; Nguyen, T.-P. Analysis of Defects and Traps in N-I-P Layered-Structure of Perovskite Solar Cells by Charge-Based Deep Level Transient Spectroscopy (Q-DLTS). J. Phys. Chem. C 2018, 122, 17601-17611.

(67) Rosenberg, J. W.; Legodi, M. J.; Rakita, Y.; Cahen, D.; Diale, M. Laplace Current Deep Level Transient Spectroscopy Measurements of Defect States in Methylammonium Lead Bromide Single Crystals. J. Appl. Phys. 2017, 122, 145701.

(68) Wei, D.; Ma, F.; Wang, R.; Dou, S.; Cui, P.; Huang, H.; Ji, J.; Jia, E.; Jia, X.; Sajid, S.; Elseman, A. M.; Chu, L.; Li, Y.; Jiang, B.; Qiao, J.; Yuan, Y.; Li, M. IonMigration Inhibition by the Cation- $\pi$ Interaction in Perovskite Materials for Efficient and Stable Perovskite Solar Cells. Adv. Mater. 2018, 30, 1707583.

(69) Yang, W. S.; Park, B.-W.; Jung, E. H.; Jeon, N. J.; Kim, Y. C.; Lee, D. U.; Shin, S. S.; Seo, J.; Kim, E. K.; Noh, J. H.; Seok, S. I. Iodide Management in Formamidinium-Lead-Halide-Based Perovskite Layers for Efficient Solar Cells. 
Science 2017, 356, 1376-1379.

(70) Yelon, A.; Movaghar, B.; Branz, H. M. Origin and Consequences of the Compensation (Meyer-Neldel) Law. Phys. Rev. B 1992, 46, 12244-12250.

(71) Metselaar, R.; Oversluizen, G. The Meyer-Neldel Rule in Semiconductors. J. Solid State Chem. 1984, 55, 320-326.

(72) Ravishankar, S.; Aranda, C.; Sanchez, S.; Bisquert, J.; Saliba, M.; GarciaBelmonte, G. Perovskite Solar Cell Modeling Using Light and Voltage Modulated Techniques. J. Phys. Chem. C 2019, 123, 6444-6449.

(73) Bertoluzzi, L.; Bisquert, J. Investigating the Consistency of Models for Water Splitting Systems by Light and Voltage Modulated Techniques. J. Phys. Chem. Lett. 2017, 8, 172-180. 